\section{Liver transplantation for alcoholic liver disease}

\author{
LOUISE HOWARD and TOM FAHY
}

transplant recipients, and no study has investigated the effect of subsequent drinking on any improvement that may occur.
End-stage alcoholic cirrhosis was first accepted as an appropriate indication for liver transplantation in the 1980s (Anonymous, 1983), though liver transplantation for patients with alcoholic liver disease (ALD) remains controversial. In 1989 ALD became the most common indication for liver transplantation in the United States (Sorrell et al, 1992), and it seems likely that increasing numbers of ALD patients will be referred to transplant units in the future. Such a trend would provoke concern among health professionals and lay persons as there are fears that these patients may return to alcohol misuse, become non-compliant and have a poor medical outcome.

The onset of liver disease can motivate alcoholics to reduce their drinking, and liver transplantation may therefore prove to be an even more potent motivating force "the ultimate sobering experience", according to Starzl, the surgeon who pioneered liver transplantation (Starzl et al, 1988). Similar survival rates are reported for ALD patients compared with other patients following liver transplantation in the short term (Startzl et al, 1988; Bird et al, 1990; Berlakovich et al, 1994), and on longer-term follow-up of up to 7-10 years (Belle et al, 1997; Pereira et al, 1997). Median followups are still relatively short and studies of outcome over more extended periods are necessary, but the promising results to date have meant that the issue for the 1990s has been less whether such patients should receive transplants but which patients should be referred for transplantation (Sherman \& Williams, 1995). Psychiatrists are being asked to participate in these decisions and therefore need to know whether it is possible to predict outcome in ALD liver transplant patients, how to advise their medical and surgical colleagues when a patient with ALD is being assessed for referral to a transplant unit, and how to treat patients when they return to local services after liver transplantation. Liaison psychiatrists attached to a transplant unit may also need to develop special services for this group of patients.

\section{MEDICAL AND FUNCTIONAL OUTCOME}

There is little difference between ALD patients and other patients post-transplantation in medical outcome, psychiatric morbidity, functional status and quality of life (e.g. Beresford et al, 1992; Howard et al, 1994a; Coffman et al, 1997). However, one recent study has suggested that by the third year post-transplantation, quality of life may be diminished in ALD patients compared with non-ALD patients (Wiesner et al, 1997); other research groups need to investigate quality of life over longer follow-up periods.

Patients transplanted for ALD have a significantly lower incidence of rejection compared with most other liver transplant patients, possibly as a result of non-specific immunological hyporesponsiveness (Berlakovich et al, 1996; Farges et al, 1996; Wiesner et al, 1997). There is little evidence of any particular problems with noncompliance in this patient group (Beresford et al, 1992; Howard et al, 1994a) and a study of resource utilisation has shown no difference in outcome between ALD and non-ALD patients undergoing liver transplantation (McCurry et al, 1992).

Alcoholic cirrhosis is associated with a similar level of cognitive impairment to that found in patients with non-alcoholic cirrhosis (Tartar et al, 1988). Cognitive function in alcoholic cirrhosis improves substantially after liver transplantation, although memory disturbance shows less improvement (Arria et al, 1991). However, clinicians may find it difficult to distinguish between cognitive impairment secondary to hepatic encephalopathy, and irreversible brain damage. There have been no controlled studies to date comparing cognitive improvement in ALD and non-ALD liver

\section{DO ALCOHOLIC LIVER TRANSPLANT PATIENTS RETURN TO DRINKING?}

Initial studies involving short follow-up periods found return to drinking in 6-23\% of ALD patients (Starzl et al, 1988; Bird et al, 1990; Beresford et al, 1992). However, longer periods of follow-up (median 33-36 months) demonstrate higher levels of exposure to alcohol, with reports of $31 \%$ (Beresford, 1994) and 32\% (Berlakovich et al, 1994) of patients failing to remain abstinent, with $15-17 \%$ returning to problem drinking. A few alcohol-related posttransplantation deaths have also been reported (e.g. Starzl et al, 1988; Berlakovich et al, 1994). The Kings College study (Howard et al, 1994a) found even higher levels of exposure to alcohol, with 19 of 20 patients admitting to some alcohol use since transplantation, eight patients regularly drinking three or more units per day, three patients drinking heavily (approximately 20 units per day) and 13 patients binge drinking (4-28 units). Patients who do return to heavy drinking develop graft damage rapidly; two patients developed cirrhosis within four years of liver transplantation (Howard et al, 1994a). A significant minority of patients $(10-15 \%$ of transplant survivors) are therefore returning to problem drinking, with many other patients remaining vulnerable to alcohol misuse.

The wide variation in the incidence of reported alcohol relapse and exposure is probably due to methodological differences between studies. Earlier studies, which included follow-up periods of less than one year, underestimated the subsequent return to drinking. Some centres have relied on patients' accounts of their alcohol intake and used investigators who have been part of the transplant team (so that patients may have felt unable to be honest about their difficulties). Most studies have reported on small numbers of patients using a cross-sectional design and failed to use standardised validated instruments of psychosocial outcome and alcohol consumption. Many researchers have not stated how much alcohol is consumed by patients who return to drinking, or how 'problem drinking' is defined. Involvement of unbiased psychiatric staff in follow-up of patients would address most of 
these shortcomings and we would recommend this approach at all transplant centres. Recent recommendations suggest that patients should be followed-up with random breath tests and testing for blood alcohol levels and serum biochemical markers for alcohol use (Hoofnagle et al, 1997). We also recommend regular discussion of alcohol exposure in primary care and at the liver transplant centre. Patients could be advised before the transplantation that these assessments will take place on follow-up, and many centres now advocate that a formal contract with the patients be signed before acceptance onto the transplant programme.

Patients undergoing transplantation for ALD are at risk of recurrence of problem drinking, and recent reports demonstrate that alcohol exposure after transplantation for ALD is associated with adverse health events (e.g. Coffman et al, 1997; Pereira et al, 1997). Long-term follow-up of male alcohol-misusing patients suggests that relapse is rare after long-term abstinence, whereas controlled drinking is usually associated with relapse (Vaillant, 1996). It therefore seems safest to advise all ALD transplant recipients to remain abstinent.

\section{PREDICTORS OF RETURN TO DRINKING}

Potential predictors of a return to alcohol misuse include length of abstinence pretransplantation, severity of alcohol dependence, social support and psychiatric morbidity. Most workers have investigated the significance of pre-operative abstinence, although findings have proved inconclusive, with some groups finding abstinence of more than six months an important predictor of abstinence post-transplantation (Bird et al, 1990; Osorio et al, 1994), whereas others have found length of abstinence to be a poor predictor (Beresford et al, 1992; Knechtle et al, 1992). These differences may reflect differences in selection criteria and validation of abstinence. Alcohol research would suggest that an arbitrary six-month period of abstinence is not a good predictor of longterm outcome (Vaillant, 1996) and some patients may be abstinent as a result of the severity of their end-stage liver disease, rather than through motivation for longterm abstinence. However, a study on the outcome of patients undergoing transplantation for alcohol hepatitis with cirrhosis (hence with periods of abstinence of less than six months) reported that only $51 \%$ of patients maintained sobriety at one-year follow-up (Bonet et al, 1993). Most centres therefore advocate a minimum six-month abstinence period before acceptance onto a liver transplantation programme.

Abstinence is also necessary to establish the benefit of an alcohol-free period on liver functioning and hence the necessity of transplantation. We would therefore recommend abstinence before accepting a patient for liver transplantation, but would not use a six-month abstinence period as an absolute exclusion criterion in patients who are committed to abstinence, are compliant with medical and alcohol treatment and have no other contraindications to transplantation. However, patients with shorter periods of pre-operative abstinence should be monitored particularly closely on follow-up.

There has been little research into other possible predictors of drinking outcome. There is evidence that most patients with ALD are less severely dependent on alcohol than patients attending alcohol clinics (Wodak et al, 1983). Retrospective assessment of dependence using a standardised selfreport questionnaire confirms that transplant patients with ALD are not a particularly dependent group (Howard et al, 1994a). The effect of severity of dependence on drinking outcome needs to be investigated further in prospective studies.

There is little evidence to suggest that social support influences outcome (e.g. Berlakovich et al, 1994), although this may reflect selection bias as most reports of alcoholic liver transplant patients are of a well-supported group of patients. Premorbid social stability and Alcoholics Anonymous attendance are important determinants of sustained abstinence in alcoholics (Vaillant et al, 1983) and on this basis it seems important to maximise support for patients (Howard et al, 1994b). Little is known of the effect of other comorbidity (e.g. psychiatric disorders, hepatitis C) on outcome, and this needs further investigation.

\section{PSYCHIATRIC ASSESSMENT}

Psychiatrists may be asked to assess the suitability of ALD patients for liver transplantation. There are marked differences between centres in the criteria used for rejection of transplantation candidates on psychosocial grounds (Collis \& Lloyd, 1992). We recommend that patients must accept the necessity of abstinence from alcohol, and compliance with all forms of treatment, but we would argue against other absolute psychosocial contraindications at this stage in our knowledge. The liaison psychiatrist is well placed to advise the transplant team on the patient's future psychiatric and social needs.

Assessment of the patient's alcohol problem should involve a thorough alcohol history from the patient and their family, with details of treatment programmes undertaken and those intended for the future. Most psychiatrists stress the importance of a commitment to abstinence, motivation, a rehabilitation relationship, substitute activities and acceptance of the diagnosis of alcoholism (Beresford, 1994). The psychiatrist can also identify, destigmatise and treat other psychiatric disorders, and ensure that these do not prevent acceptance of a patient onto a transplantation programme; in our experience, vague references to personality disorder or affective disorder can be seen as absolute contraindications to transplantation, even when the transplant team do not have details of a patient's previous functional status, compliance or psychiatric prognosis.

Prediction of drinking behaviour posttransplantation in any individual is limited, but psychiatrists can identify potential difficulties and advise on their treatment (e.g. mobilising support from the family, treating comorbid psychiatric disorder and arranging adequate treatment of the alcohol problem post-transplantation). Psychiatrists can also make an independent assessment of competence to consent to transplantation. The provision of psychiatric assessments for all alcoholics on liver transplant units could have major resource implications for liaison psychiatry services, as this work is time-consuming, complex and needs to be provided quickly (Mitchell et al, 1997), particularly if patients are deteriorating medically. However, dedicated psychiatric sessions attached to each transplant programme are essential for full assessment and treatment of these patients.

\section{TREATMENT AFTER TRANSPLANTATION}

ALD patients do not have a higher incidence of post-operative psychiatric problems than non-ALD patients (Beresford, 1994; Howard et al, 1994a) but psychiatric expertise may be required by the transplant 
team to help in the management of postoperative delirium or non-organic psychiatric disorders. It is less clear how ALD patients should be treated in the long term. Should all liver transplant patients with a history of ALD be followed up by a psychiatrist with an interest in addictions for the rest of their lives? Should they be seen by the psychiatrist attached to the liver transplant unit (which would mean more dedicated resources for this service) or should patients be sent back to local services? Alternatively, a liaison psychiatrist could ensure that adequate followup has been arranged after some initial input during the assessment period and during the in-patient stay after transplantation.

It may not be realistic to place every ALD patient in an alcohol treatment programme. Treatments should then be targeted to patients who are at increased risk of drinking or who have resumed drinking (Beresford, 1994). However, the cost of psychiatric interventions is likely to be low compared with the large financial investment involved in the high technology of the liver transplantation itself. Randomised treatment interventions are needed in prospective multi-centre trials to assess the optimal management of these patients after transplantation. We would recommend that at present all patients transplanted for ALD are seen before discharge by the liaison psychiatrist for an assessment of their treatment needs, and that a treatment plan is handed over to the general practitioner or local psychiatric services with explicit suggestions on engagement with voluntary or statutory services to maintain abstinence.

\section{ETHICAL ISSUES}

Outcome in ALD patients has proved to be better than expected in the early days of liver transplantation, but these patients remain vulnerable to physical and psychological problems. Members of the public and health professions may feel that in view of the limited number of liver transplants available, ALD patients should not be given liver transplants or at least not compete equally with other, more 'deserving' patients, as the liver disease may be viewed as the patient's own fault. Similar arguments have recently been aired in the media after it was erroneously alleged that a 15-yearold girl with acute liver failure was refused a liver transplant on the moral grounds that she had experimented with drugs (The
Times, 24 January 1997, p. 1). Others have argued that the inclusion of patients with ALD in liver transplantation programmes may lead to a lowering of public support, with a consequent decrease in the number of donor livers (Moss \& Siegler, 1991).

Questions of individual responsibility are not clear-cut: genetic, social and legal factors are involved in the aetiology of alcoholism. Patients with a higher risk of relapse of liver disease (e.g. patients with hepatomas or hepatitis B) are transplanted without similar censure. Similarly, patients with other 'self-inflicted' diseases such as lung cancer related to smoking are treated by the medical profession without hesitation. Organ availability is limited but better education of the public on the good outcome of 'former alcoholics' could lead to more organ donations (Schenker et al, 1990). The research to date suggests that ALD is not a medical contraindication to liver transplantation, and discrimination against this group of patients would therefore be due to an assessment of worthiness which should be made by society rather than by individual clinicians. These ethical considerations need to be considered by all transplantation centres; pre- and posttransplantation psychiatric evaluation addresses most of these pertinent ethical issues.

\section{CONCLUSIONS}

Patients transplanted for alcoholic liver disease have similar medical outcomes to those transplanted for other liver diseases. These patients remain vulnerable to alcohol misuse, although little is known about predictors of alcohol relapse in this group. Liaison psychiatrists can help identify potential post-operative problems and ensure adequate support is available if problems arise. In view of the high costs and increasing level of demand for such services, we recommend that all transplant units incorporate psychiatric services into their routine procedures and costs. Prospective studies are essential and should include randomised psychosocial interventions in order to assess the optimal management of alcoholic liver transplant patients.

\section{REFERENCES}

Anonymous (1983) Liver transplantation. Journal of the American Medical Association, 250, 2961-2964.

Arria, A. M., Tartari, R. E., Starzl, T. E., et ol (1991) Improvement in cognitive functioning of alcoholics following or thoptic liver transplantation. Akohol: Clinical and Experimental Research, 15. 956-962.

Belk, S. H., Beringer, K. C. \& Detre, K. M. (1997) Liver transplantation for alcoholic liver disease in the United States: 1988 to 1995. Liver Transplantation and Surgery. 3. $212-219$

Beresford, T. P. (1994) Psychiatric follow-up care of alcoholdependent liver graft recipients. In Liver Transplantotion and the Alcoholic Patients (eds M. R. Luceny, R. M. Merion \& T. P. Beresford). pp. 96-112. Cambridge: Cambridge University Press.

—, Schwartz, J., Wilson, D., et al (1992) The short term psychological health of alcoholic and non-alcoholic liver transplant recipients. Alcohol: Clinical and Experimental Research. 16. 996-1000.

Berlakovich, G. A., Steininger, R., Herbst, F., et ol (1994) Efficacy of liver transplantation for alcoholic cirrhosis with respect to recidivism and compliance. Transplantotion, 58. 560-565.

_, Imhof, M., Karner-Hanusch, J., ot of (1996) The importance of the effect of underlying disease on rejection outcomes following or thoptic liver transplantation. Transplantotion, 61, 554-560.

Bird, G. L. A., O'Grady, J. G., Marvey, F. A. H., et al (1990) Liver transplantation in patients with alcoholic cirrhosis: selection criteria and rates of survival and relapse. British Medical journal, 301. 15-17.

Bonet, H., Manez, R., Kramer, D., ot ol (1993) Liver transplantation for alcoholic liver disease: survival of patients transplanted with alcoholic hepatitis plus cirrhosis as compared to those with cirrhosis alone. Akohol: Clinical and Experimental Reseorch. 17. 1102-1109.

Coffman, K. L., Hoffman, A., Sher, L., ee al (1997) Treatment of the postoperative alcoholic liver transplant recipient with other addictions. Liver Tronsplontation and Surgery, 3, 322-327.

Collis, I. \& Lloyd, G. (1992) Psychiatric aspects of liver disease. British journal of Psychiatry, 161, 12-22.

Farzes, O., Salliba, F., Farhamant, H., ef of (1996) Incidence of rejection and infection after liver transplantation as a function of the primary disease: possible influence of alcohol and polyclonal immunoglobulins. Hepotology, 23. 240-248.

Hoofnagle, J. H., Kresina, T., Fuller, R. K., et ol (1997) Liver transplantation for alcoholic liver disease: executive statement and recommendations. Liver Transplantation and Surgery. 3, 347-350.

Howard, L. M., Fahy, T., Wong, P., et ol (1994a) Psychiatric outcome in alcoholic liver transplant patients. Quarterly journal of Medicine, 87. 731-736.

_, Williams, R. \& Fahy, T. A. (19946) The psychiatric assessment of liver transplant patients with alcoholic liver disease: a review. Journal of Psychosomatic Research, 38. 643-653.

Knechtle, S. J., Fleming, M. F., Barry, K. L., et ol (1992) Liver transplantation for alcoholic liver disease. Surgery, $I 12$. 694-703.

McCurry, K. R., Ballza, P., Merion, R. M., et al (1992) Resource utilization and outcome of liver transplantation for alcoholic cirrhosis - a case control study. Archives of Surgery. 127. 772-777.

Mitchell, J. R., Lang, F. H. \& Masterton, G. (1997) Providing a psychiatric service to liver transplant patients. The first 2 years of the Scottish Liver Transplant Unit. Psychiatric Bulletin, 21. 6-9.

Moss, A. H. \& Siegler, M. (1991) Should alcoholics compete equally for liver transplantation? Journol of the American Medical Associotion. 265. 1295-1298. 
Osorio, R. W., Ascher, N. L., Avery, M., et ol (1994)

Predicting recidivism after or thoptic liver transplantation

for alcoholic liver disease. Hepotology. 20. 105-110.

Pereira, S. P., Mulesan, P., Howard, L. M., et ol (1997) Long-term survival and alcohol relapse after liver

transplantation for alcoholic liver disease. Gut. $\mathbf{4 0}$ (suppl. I). Al8

Schenker, S., Perkins, H. S. \& Sorrell, M. F. (1990) Liver transplantation in alcoholics. Hepotology. II. 314-319.

Sherman, D. Williams, R. (1995) Liver transplantation for alcoholic liver disease. Journal of Hepatology, 23. 474-479.

Sorrell, M. F., Donovan, J. P. Shaw, B. W. (1992)

Transplantation in the alcoholic: a stalking horse for a larger problem. Gastroenterology. 102. 1806- 1808.

Starzl, T. E., Van Thiel, D., Tzakis, A. G., et ol (1988)

Or thoptic liver transplantation for alcoholic cirrhosis. Journal of the American Medical Association, 260. 2542-2544.

LOUISE HOWARD. MRCPsych, Department of Psychological Medicine, Kings College Hospital, Bessemer Road, London; TOM FAHY, MRCPsych, Maudsley Hospital, Denmark Hill, London

Correspondence: Dr Louise Howard, Department of Psychological Medicine, Kings College Hospital, Bessemer Road, London SE5 9RS

(First received 4 March 1997, revised I August 1997, accepted 26 August 1997)

Tarter, R. E., Van Thiel, D., Arria, A. M., et al (1988) Impact of cirrhosis on neuropsychological test performance of alcoholics. Alcoholism, 12, 619-621.

Vaillant, G. E. (1996) A long-term follow-up of male alcohol abuse. Archives of General Psychiatry. 53. 243-249.

_. Clark, W., Cyrus, C., et al (1983) Prospective study of alcoholism treatment. American journal of Medicine, 75. $455-463$.
Wiesner, R. H., Lombardero, M., Lake, J. R., et ol (1997) Liver transplantation for end-stage alcoholic liver disease: an assessment of outcomes. Liver Transplantation and Surgery. 3. 231-239.

Wodak, A. D., Saunders, J. B., Ewusi-Mensah, I., et af (1983) Severity of alcohol dependence in patients with alcoholic liver disease. British Medical Journal, 287. 1420-1422 